\title{
Basic Molecular and Cell Biology
}

\section{An introduction to cells}

\author{
L WOLPERT
}

Cells are the triumph of evolution. ${ }^{1}$ The rest of evolution can be thought of as an elaboration on this masterpiece. In some ways they are more complex than the organs to which they give rise, with the possible exception of the brain, in that their behaviour reflects the integrated activity of about 50000 genes, their products, and the complex biochemical and structural networks that result.

In this biochemical network there are two different timescales. The first, which responds to changes in seconds or fractions of a second, is that concerned with metabolism. The enzymes in the cell cytoplasm, including the mitochondria, catalyse molecules along narrowly defined reaction pathways such as the Krebs cycle, the synthesis of purines, or the breakdown of carbohydrates. Many of these reactions require or generate energy in the form of adenosine triphosphate. The speed of response of these metabolic pathways can be contrasted with those in the second system, which entails the synthesis of macromolecules such as nucleic acids and proteins. Here the response times are minutes to hours. Understanding the integration of these two interdependent pathways is a major problem in cell biology.

\section{Proteins characterise cells}

The character of a cell is determined by the proteins it contains. For example, the special feature of parenchymal liver cells is that they synthesise albumin, whereas lymphocytes synthesise globulin. The enzymes in a cell determine its metabolic pathways. The control of protein synthesis is thus central to the life of the cells and is controlled by nuclear cytoplasmic interactions.

The genes on the chromosomes in the cell nucleus dominate the life of the cell by controlling protein synthesis. ${ }^{2}$ But they are curiously passive. The deoxyribonucleic acid (DNA) of the genes codes only for proteins or for ribonucleic acid (RNA) itself. Typically, a DNA sequence is transcribed into a messenger RNA sequence, which is then processed within the nucleus, non-coding sequences known as introns being removed. It is this processed RNA that enters the cytoplasm as messenger RNA, where it is translated into the amino acid sequence of the protein on ribosomes. Much of the RNA transcribed within the nucleus never reaches the cytoplasm, and this is an important level of control in the nucleus in addition to the control of transcription itself. The genetic control of cell activity is entirely the result of the control of protein synthesis, and thus the genes do not exert an immediate effect on the life of the cell. If the nucleus is removed from the cell the metabolic pathways are unaffected until there is a change in enzyme concentration. This will depend on both the stability of the proteins and their messenger RNA.

\footnotetext{
Department of Anatomy and Biology as Applied to Medicine, Middlesex Hospital Medical School, London W1P 6DB

L WOLPERT, FRS, professor
}

\section{DNA and function and form}

Since there is good evidence that the DNA is the same in almost all cells in the body of an individual, there must be mechanisms which turn genes on and off in specific cells. Development can thus be viewed in terms of the control of differential gene activities, DNA providing a genetic programme. As development proceeds different cell types with different protein compositions emerge. Not only must genes be turned on and off, but also this activity must be stable so that it can be inherited when the cells multiply. Liver cells divide to give more liver cells.

Changes in DNA can alter cell behaviour. Mutations alter the sequence of the bases. This is what happens in sickle cell anaemia, where the haemoglobin molecules have abnormal properties. A change in one of the codons of the gene for haemoglobin alters one amino acid, glutamine, to valine. This in turn changes the way the molecule folds and results in the haemoglobin molecules sticking together and forming rod like structures, which deform the red cell, giving it its sickle shape. This alters the flexibility of the cell and so affects its passage through fine capillaries, which results in anaemia. Thus from a change in just one base there is a cascade of events leading to a physiologically abnormal condition.

Sickle cell anaemia is an excellent example of the relation between DNA and function and form. In contrast to the intimate relation between DNA and protein, the pathways whereby a protein affects the working of the cell and the body as a whole can be intricate, tortuous, and multifarious. In general these pathways are not known, and this is a major problem in cell biology. Even if we knew the complete sequence of the DNA of human cells we could not, at present, interpret it.

\section{Role of cytoplasm}

A central problem is what controls the transcription of particular genes. This clearly entails cytoplasmic factors, probably proteins, but they have proved difficult to isolate. It is presumed that such factors act on the promoters adjacent to particular structural genes and allow them to be transcribed in specific tissues. It is possible to attach the growth hormone gene (normally active only in the pituitary) to the elastase promoter (elastase is made only in the pancreas). When this construction is incorporated into the genome by injecting the DNA into the nucleus of a fertilised mouse egg growth hormone is made in the pancreas.

Further evidence for cytoplasmic signals comes from experiments in which cells are fused so that their nuclei are in a mixed cytoplasm. Under such conditions human cancer cell cytoplasm can reactivate the dormant nucleus of the chick red blood cell. This technique of cell fusion has another and even more important application: it provides the basis of human somatic cell genetics. When mouse and human cells are fused and the cells allowed to proliferate, human chromosomes are lost. This allows particular functions to be assigned to particular chromosomes.

The different proteins must be transported to the specific cellular compartments where they will be used: enzymes of the Krebs cycle 
to the mitochondria and degradative enzymes to liposomes. This is achieved by the extensive membrane bound system in the cytoplasm, particularly the endoplasmic reticulum and the Golgi apparatus. To achieve this sorting specific amino acid sequences serve as address markers. For example, if the amino acid terminal end carries the so called signal sequence the proteins are transferred across the membrane of the endosplasmic reticulum. Those that do not have the sequence remain in the cytosol. Further sorting then occurs so that, for example, some of those that enter the endoplasmic reticulum go via the Golgi apparatus to the plasma membrane or special secretory vesicles. It is clear that there is considerable organisation of the internal membrane system in the cytoplasm.

A major change in our concept of the cell relates to the structure of the cytoplasm or, more fashionably, the cytoskeleton. A substantial fraction of the cellular proteins make up a fibrous network, which seems to serve as a scaffold. This scaffold organises many other cell constituents and is concerned with both maintaining and altering the shape of the cell and with bringing about cell movement. Major components here are actin filaments, microtubules, and intermediate filaments. Intermediate filaments are stable compared with microtubules and actin filaments, which are continually assembling and disassembling. There is a large research industry devoted to analysing this process, particularly in relation to motility and the role of other proteins. There is also a major effort towards understanding how the cytoskeleton is linked to the plasma membrane. One view is that events at the surface may be transduced via the cytoskeleton to provide a signal to the cell nucleus. This is closely linked with the recent emphasis given to the possible importance of cell shape and the role of the extracellular matrix in determining cell behaviour.

\section{Extracellular matrix and plasma membrane}

Rather than being perceived just as rather dull packing the extracellular matrix, which is secreted by the cells, is now seen as having a major role in determining cellular behaviour. This is particularly true of epithelia. For example, mammary gland epithelium in culture behaves quite differently depending on the nature of the matrix to which it is attached. This interaction may be mediated by the matrix determining cell shape. Again, the migration of cells is particularly dependent on the matrix. Major matrix components such as fibronectin have been identified only quite recently, and the classes of collagens seem to increase annually.

The plasma membrane is now considered in terms of a fluid bimolecular lipid membrane in which proteins, such as receptors, float, move, and interact. Attention is focused on transmembrane events mediated by these proteins. For example, some growth factors are thought to bind to receptors which activate a phospholipase on the inner surface. This splits phospholipid into inisitoltriphosphate and diacyglycerol. The former leads to an increase in calcium ions, while the latter activates phospholipase. The remainder of the pathway is largely unknown.

It is remarkable that with the exception of steroid hormones we do not know the sequence of events that lead from an external signal to the cell's response. The sequence for glucocorticoid is well established: it enters the cell, binds a cytoplasmic protein, enters the nucleus, and attaches to specific sequences of DNA activating genes. ${ }^{3}$ But in all other cases, including the action of insulin, growth hormones, and adrenaline, for which some of the early steps are known, the sequence peters out. Cells are complex little organisms. This is particularly dramatically illustrated by oncogenes such as src. We know that the cell is transformed into a malignant cell by this gene-the sequence is fully known. But how does it do it? The gene codes for a protein kinase, but the sequence of cascade like events is simply not known.

Cells are not what they used to be. Their study has been transformed by new techniques, particularly the analysis of cell components in molecular terms and the all persuasive influence of recombinant DNA technology. Remarkable progress has been made in decomposing and reassembling cellular structures and functions in vitro. The most recent is the ability to reform nuclei around injected DNA in a cell free extract. ${ }^{4}$ Perhaps the assembly of whole and new kinds of cells is not too far off. Will our grandchildren have 48 chromosomes?

\section{References}

1 Alberts B, Bray D, Lewis J, Raff M, Roberts K, Watson JD. The molecular biology of the cell. London: Garland, 1983.

2 Wolpert L. DNA and its message. Lancet 1984;ii:853-6.

3 Yamamoto KR. Steroid receptor regulated transcription of specific genes and gene networks. Annu Rev Genet 1985; 19:209-52.

4 Fisher PA. Disassembly and reassembly of nuclei in cell free systems. Cell 1987;48:175-6.

(Accepted 20 May 1987)

\section{MATERIA NON MEDICA}

\section{Crumbs and coffee stains}

The doctor, a psychiatrist, returned to speak to the patient's wife. This was immediately after having afternoon tea with her to discuss her husband's problem of amnesia following a war injury. On approaching the tea table he picked up the biscuit that he had not eaten the first time round. Promptly the lady handed him the empty plate so that the doctor would not strew the biscuit crumbs all over the expensive carpet in the drawing room of the stately home. The doctor was Ian Holm, the wife was Julie Christie, and Alan Bates the amnesic patient needing psychiatric help-all this in a television play based on a novel by Rebecca West.

My wife chuckled at this incident, which had at once reminded me of what goes on in doctors' sitting rooms in hospitals all over the United Kingdom. Why is it that all sitting rooms in doctors' quarters are in a perpetually filthy state except perhaps for an hour just after they have been cleaned? You need only to enter the sitting room first thing in the morning to see the mess-half empty tankards of stale beer, recumbent beer cans or Guinness bottles partly emptied on the carpet, innumerable coffee cups, most of them used without their saucers so as to leave their tell tale stains on the table, saucers used as ash trays, the odd foil tray from a Chinese take away, newspapers strewn all over the place, and on the corner table scattered playing cards, the legacy of the intellectuals, bridge/poker players-all to be cleared away and cleaned up by the domestic.

Now that an adequate pantry is usually attached to operating theatres and even to a few doctors' sitting rooms in the newer hospitals it would not go amiss if the users washed their cups (and saucers) and left them in a condition fit to be used by their colleagues later on. No, some wouldn't do that because they fill their cups with ash from their pipes or cigarettes-and this despite a nearby empty ash tray-thus making a concoction of left over coffee and ash to be cleaned by the unfortunate domestic whose comments I shall leave to the readers' imagination. Some years ago, in an operating theatre during the mid-morning coffee break, all the cups had been used up by the time one of the junior doctors was the last in for coffee (he must have been an anaesthetist); not finding a single clean cup he asked the sister if she could order some more clean cups, to which came the obvious reply that we were all waiting for: "Wash one of those that are there"-an obvious solution to the problem. The doctor in question being from the subcontinent, the land of the erstwhile maharajahs, and therefore perhaps used to servants, such a solution had simply not occurred to him.

Filthy doctors' sitting rooms are here to stay and be tolerated by the silent minority (majority?). The director of the play, which was a post first world war piece, obviously depicted the doctor as belonging to the modern erahaving undisciplined eating and drinking habits.

Ugh! as I complete this piece, my coffee mug has produced a stain on the journal used to protect my desk; the journal in question: the $B M \mathcal{F}-$ well, the classified advertisement section to be precise. I am not looking for a job in the near future-not yet, anyway.-PRADIP K DATTA, consultant surgeon, Wick, Scotland. 